Pacific Journal of Mathematics

UNIFORM DIMENSIONS AND SUBDIRECT PRODUCTS 


\title{
UNIFORM DIMENSIONS AND SUBDIRECT PRODUCTS
}

\author{
JOHN DAUNS
}

Over a ring $R, \mathbf{E}$ denotes injective hulls, $\mathbf{Z}$ singular submodules. GENERAL THEOREM. For any ring $R$ and any infinite regular cardinal $\sigma$, conditions (1), (2), and (3) are all equivalent: (1) Any direct sum of nonsingular right ideals of $R$ contains fewer than $\sigma$ nonzero summands. (2) If $\left\{W_{\gamma} \mid \gamma \in \Gamma\right\}$ is any indexed set of modules with all $\mathbf{Z} W_{\gamma}=0$, then the submodule $\Pi^{\sigma} \mathbf{E}\left(W_{\gamma}\right)=\left\{x=\left(x_{\gamma}\right) \in \Pi \mathbf{E}\left(W_{\gamma}\right) \mid\right.$ support $\left.x \mid<\sigma\right\}$ is injective. (3) For any family $\left\{W_{\gamma} \mid \gamma \in \Gamma\right\}$ having all $\mathrm{Z} W_{\gamma}=0$, the submodule $\Pi^{\sigma} W_{\gamma} \leq \Pi W_{\gamma}$ is a complement. COROLLARY. Every ring $R$ satisfies (1), (2), and (3) for a unique smallest infinite regular cardinal $\sigma=\sigma(R)$. THEOREM. For any module $M$ with $\mathbf{Z} M=0, \mathbf{E}(M)=C \oplus D$, where $C$ contains no uniform submodules and $D=\Pi\left\{\mathbf{E}\left(D_{\tau}\right) \mid \tau \in \Xi\right\}$. The submodules $C, D$, and the $D_{\tau}$ are all unique. Each $D_{\tau}$ is $a$ direct sum of isomorphic indecomposable injectives all of the same type $\tau$. $\square$ The cardinal number of such summands of $D_{\tau}$ is the $\tau$-dimension of $M$. More general uniform dimensions are constructed for arbitrary modules.

1. Introduction. For any unital right $R$-module $M$, its injective hull $\hat{M}$ is uniquely up to isomorphism of the form $\hat{M}=\mathbf{Z}_{\mathbf{2}} \hat{M} \oplus C \oplus D$, where $C$ is continuous, and $D$ discrete (Theorem 1). If $\mathbf{Z} M$ is the singular submodule, then the torsion submodule $\mathbf{Z}_{2} M$ of $M$ is defined by $\mathbf{Z}[M / \mathbf{Z} M]=\left(\mathbf{Z}_{\mathbf{2}} M\right) / \mathbf{Z} M$.

Now let $M$ be torsion free, i.e., $\mathbf{Z} M=0$. A type $\tau$ is an equivalence class of nonsingular uniform modules all having isomorphic injective hulls, and $\Xi=\{\tau\}$ is the set of all types. Then $M_{\tau} \equiv \sum\{U \mid U<M$, $U \in \tau\} \leq M$ is an intrinsic submodule of $M$. The $\tau$-dimension of $M$ is the cardinality of the nonzero summands in any maximal direct sum of submodules of $M_{\tau}$.

More general dimensions $\Omega-d M$ and corresponding intrinsic submodules $M_{\Omega}$ are studied, where $\Omega$ is a disjoint union of types. Special important cases are $\Omega=\tau$ a single type, and at the other extreme, $\Omega=\Sigma$, the class of all torsion free uniform modules.

A result of Teply ([T1; p. 443, Theorem 1.2] and [T2; p. 451, Theorem 2.1]) says that every $\boldsymbol{\aleph}_{0}$-product ( $\equiv$ direct sum) of torsion free injectives is injective if and only if the Goldie dimension of $R /\left(\mathbf{Z}_{2} R\right)$ is finite. Here the analogue for higher cardinals $\sigma \geq \boldsymbol{\aleph}_{0}$ is given in Theorem V. Counterexamples 4.3 show that a complete generalization is impossible. 
In Theorem IV, the hypothesis (1) of the General Theorem II is replaced by the condition $\left(1^{\prime}\right) \tau-d R<\sigma$. Even in the finite case when $\boldsymbol{\sigma}=\boldsymbol{\aleph}_{0}$, the results are new. Then it is an immediate corollary of Theorem IV (with $\Omega=\tau$ and $\sigma=\aleph_{0}$ ) that the following conditions (1), (2), and (3) are all equivalent:

(1) $\tau-d\left(R / \mathbf{Z}_{2} R\right)$ is finite.

(2) Every direct sum of torsion free indecomposable injective modules of type $\tau$ is injective.

(3) Every direct sum of torsion free uniform modules of type $\tau$ is a complement submodule in their full direct product.

1. Preliminaries. Standardized terminology, notation, symbols, as well as some facts used throughout the rest of this note are all given at once.

1.1. Notation. Modules will be right unital over a ring $R$. Right $R$-submodules are denoted by " $<$ " essential or large ones by " $<<$ " (the same symbol as for submodules-except twice as large); “ $\triangleleft$ " denotes ideals in any ring. In $\subset, \supset,<,<<$, and $\triangleleft$ equality is also allowed. However, where important, or for emphasis, sometimes also $\subseteq$, , and $\leq$ will be used. Slashes through a symbol denote its negation: $\not \subset$, $\nsupseteq$, $<\nless$, and $\notin$. In particular " $A<\nless B$ " means that $A<B$ but that $A$ is not a large submodule of $B$.

If $M$ is a module and $\varnothing \neq Y \subseteq M$ a subset, define $Y^{\perp}=\{r \in R \mid \forall y$ $\in Y, y r=0\}=\{r \mid Y r=0\}<R$. In particular, $M^{\perp} \triangleleft R$. If $m \in M$ and $K<M$, set $m^{\perp}=\{m\}^{\perp}<R$, and for $m+K \in M / K$, define $m^{-1} K=$ $(m+K)^{\perp}=\{r \in R \mid m r \in K\}<R$. Note that for $a \in R,(m a)^{-1} K=$ $a^{-1} m^{-1} K$, but only $a(m a)^{-1} K \subseteq m^{-1} K$.

Injective hulls of right $R$-modules are denoted by “ $\wedge$ " as well as "E", e.g. $\hat{M}=\mathbf{E} M=\mathbf{E}(M)$, where $\mathbf{E}$ will be used when $M$ is given by a formula.

1.2. Singular submodules. The singular and second singular submodules of $M$ are $\mathbf{Z} M=\left\{m \in M \mid m^{\perp}<<R\right\} \leq M$ and $\mathbf{Z}[M / \mathbf{Z} M]=$ $\left(\mathbf{Z}_{\mathbf{2}} M\right) /(\mathbf{Z} M)$, where (a) $\mathbf{Z}\left[M / \mathbf{Z}_{\mathbf{2}} M\right]=0$. Also write $\mathbf{Z} M=\mathbf{Z}(M)$ and $\mathbf{Z}_{\mathbf{2}} M=\mathbf{Z}_{\mathbf{2}}(M)$. To avoid confusing a nonsingular module $(\mathbf{Z} M=0)$ with a not singular module (only $\mathbf{Z} M \neq M$ ), $M$ will be called torsion free if $\mathbf{Z} M=0$, and torsion if $\mathbf{Z}_{\mathbf{2}} M=M$. Although the functor $\mathbf{Z}$ does not give a hereditary torsion theory, $\mathbf{Z}_{\mathbf{2}}$ does, and $\mathbf{Z}_{\mathbf{2}} M=0 \Longleftrightarrow \mathbf{Z} M=0$. Furthermore, for $M=R$, (b) $\mathbf{Z}_{\mathbf{2}} R \triangleleft R$. Since (c) $M \mathbf{Z}_{\mathbf{2}} R \subseteq \mathbf{Z}_{\mathbf{2}} M$, if (d) $\mathbf{Z} M=0$, then $M\left(\mathbf{Z}_{\mathbf{2}} R\right)=0$. 
The properties of $\mathbf{Z}_{2}$ used repeatedly throughout here are that (e) $\mathbf{Z} M<<\mathbf{Z}_{\mathbf{2}} M$ and that (f) $\mathbf{Z}_{\mathbf{2}} M \leq M$ is the unique smallest complement submodule of $M$ containing $\mathbf{Z} M$.

In particular, (g) $\mathbf{Z}_{\mathbf{2}}(\mathbf{E} M)=\mathbf{E}(\mathbf{Z} M)$ is a direct summand of $\mathbf{E} M$. Also if (h) $\mathbf{Z} R=0$, then $\mathbf{Z} M=\mathbf{Z}_{\mathbf{2}} M$.

The symbols $<,<<,<\nless,{ }^{\perp},{ }^{-1}, \wedge, \mathbf{E}, \mathbf{Z}$, and $\mathbf{Z}_{\mathbf{2}}$ always refer to right $R$-submodules, and never ever to other rings, e.g. those derived from $R$ such as $R / \mathbf{Z}_{\mathbf{2}} R$, or $R / \mathbf{Z} R$, or $\mathbf{E}\left(R / \mathbf{Z}_{\mathbf{2}} R\right)$.

1.3. Cardinals. The cardinality of any set is denoted by "| |". The successor cardinal of any cardinal whatever such as $|X|$ is denoted by $|X|^{+}$, e.g. $\boldsymbol{\kappa}_{\alpha}^{+}=\boldsymbol{\aleph}_{\alpha+1}$. An infinite cardinal $\kappa$ is regular if and only if it is not a sum (i.e. a disjoint union) of the form $\kappa=\sum\left\{\kappa_{i} \mid i \in I\right\}=$ $\bigcup\left\{\kappa_{i} \mid i \in I\right\}$, where $|I|<\kappa$ as well as $\kappa_{i}<\kappa$ for all $i \in I$. (See [HJ; p. $161-163 ; 3.1,3.2]$.)

1.4. A sum of submodules of some bigger module is denoted by " $\Sigma$ ", whereas both an internal and an external direct sum will be written as “ $\oplus$ ". For modules $\left\{W_{\gamma} \mid \gamma \in \Gamma\right\}$, form $\oplus\left\{W_{\gamma} \mid \gamma \in \Gamma\right\}=\oplus W_{\gamma}<$ $\Pi\left\{W_{\gamma} \mid \gamma \in \Gamma\right\}=\Pi W_{\gamma}$. Whenever an index set is omitted, not only as above in $\oplus$ and $\Pi$, but also in $\Sigma, U$, or $\cap$, it will then be understood that the index ranges over the largest possible index set, and never over some proper subset. For $x=\left\{x_{\gamma} \mid \gamma \in \Gamma\right\}=\left(x_{\gamma}\right)_{\gamma \in \Gamma}=\left(x_{\gamma}\right) \in \Pi W_{\gamma}$, and for $Y \subset \Pi W_{\gamma}$, define their supports to be the subsets supp $x=\left\{\gamma \in \Gamma \mid x_{\gamma} \neq\right.$ $0\}$ and $\operatorname{Supp} Y=\bigcup\{\operatorname{supp} y \mid y \in Y\}$ of $\Gamma$. Thus $\operatorname{Supp}\{x\}=\operatorname{supp} x$.

1.5. Definition. For an infinite cardinal $\sigma$, the $\sigma$-product of an indexed set of sets $W_{\gamma}, \gamma \in \Gamma$, modules, or rings is defined to be the subdirect product $\Pi^{\sigma}\left\{W_{\gamma} \mid \gamma \in \Gamma\right\}=\left\{x \in \Pi W_{\gamma}|| \operatorname{supp} x \mid<\sigma\right\} \equiv \Pi^{\sigma} W_{\gamma}$. Thus for $\sigma=\boldsymbol{\aleph}_{0}=|\omega|=\left|\omega_{0}\right|, \Pi^{\sigma}$ is just the ordinary direct sum.

1.6. For an infinite cardinal $\sigma$ and modules $W_{\gamma}, \gamma \in \Gamma$, and one single element $x=\left(x_{\gamma}\right) \in \Pi W_{\gamma}$, define $A_{i}<R, i \in I$ as follows: $I=\{i \subset$ $\Gamma|| I \mid<\sigma\}, A_{i}=\bigcap\left\{x_{\gamma}^{\perp} \mid \gamma \notin i\right\}$. Note that also $A_{i}=\bigcap\left\{x_{\gamma}^{\perp} \mid \gamma \in \operatorname{supp} x\right.$; $\gamma \notin i$. Hence Supp $x A_{i} \subseteq i$, and $A_{i}$ is the unique largest right ideal of $R$ with this property. If $i \subset j \in I$, then $A_{i} \subseteq A_{j}$.

If $J, K \subseteq I$ are any subsets, then $\bigcap\left\{A_{k} \mid k \in K\right\}=A_{\cap K}$, where $\bigcap K=\bigcap\{k \mid k \in K\} \in I$, whereas $\sum\left\{A_{j} \mid j \in J\right\} \subseteq A_{\cup J}$, provided that $\cup J$ $\in I$, i.e. that $|\cup J|<\sigma$. Consequently, for $x+\Pi^{\sigma} W_{\gamma} \in \Pi W_{\gamma} / \Pi^{\sigma} W_{\gamma}$, $\left(x+\Pi^{\sigma} W_{\gamma}\right)^{\perp}=x^{-1} \Pi^{\sigma} W_{\gamma}=\bigcup\left\{A_{i} \mid i \in I\right\}=\sum A_{i}$. 
2. Types and Uniform Dimensions. First it is shown that any injective module $M$ can be decomposed as a direct sum $M=C \oplus C^{\prime} \oplus$ $D^{\prime} \oplus D$ of four different kinds of modules; $C \oplus C^{\prime}$ is continuous or uniform free; $D^{\prime} \oplus D$ is discrete, that is contains an essential direct sum of uniform submodules; $C^{\prime} \oplus D^{\prime}$ is torsion, while $C \oplus D$ is torsion free.

2.1. Lemma. Every injective module $M$ is uniquely of the form $M=F \oplus$ $G$ up to isomorphism, where $G$ contains an essential direct sum of uniform modules and $F$ is uniform free.

Proof. Zorn's lemma applied to the set of sets of uniform submodules whose sum is direct produces $M=F \oplus G$. Similarly, let also $M=F_{1} \oplus$ $G_{1}$. By the common refinement property ([W; p. 268, Cor. 1.1]), decompose further $F=F^{\prime} \oplus F^{\prime \prime}, G=G^{\prime} \oplus G^{\prime \prime}$, etc. so that

$$
\begin{aligned}
& M=F^{\prime} \oplus F^{\prime \prime} \oplus G^{\prime} \oplus G^{\prime \prime}=F_{1}^{\prime} \oplus F_{1}^{\prime \prime} \oplus G_{1}^{\prime} \oplus G_{1}^{\prime \prime}, \text { where } \\
& F^{\prime} \cong F_{1}^{\prime} ; \quad G^{\prime \prime} \cong G_{1}^{\prime \prime} ; \quad F^{\prime \prime} \cong G_{1}^{\prime} ; \quad \text { and } \quad G^{\prime} \cong F_{1}^{\prime \prime} .
\end{aligned}
$$

Thus $F^{\prime \prime}=G_{1}^{\prime}=0$ and $G^{\prime}=F_{1}^{\prime \prime}=0$. Hence $F=F^{\prime} \cong F_{1}^{\prime}=F_{1}$, and $G=G^{\prime \prime} \cong G_{1}^{\prime \prime}=G_{1}$.

2.2. Definition. Modules $A$ and $B$ are parallel-written as $A \| B$-provided that $A$ does not contain a nonzero submodule isomorphic to some submodule of $B$.

2.3. Lemma ([D4; p. 34, Theorem II]). Let $A$ and $B$ be submodules of some bigger module, and let $C$ be any torsion free module with $A \| C$ and $B \| C$. Then also likewise $(A+B) \| C$.

2.4. Lemma. Let $\left\{A_{\alpha}\right\}$ be any set of submodules $A_{\alpha}<C$ of $C$ and $\left\{B_{\beta}\right\}$ another set of submodules $B_{\beta}<D$ of some module $D$. Assume that $\mathbf{Z C}=0$; and that for all $\alpha$ and $\beta, \mathbf{Z} B_{\beta}=0$, and $A_{\alpha} \| B_{\beta}=0$. Then

(i) $\sum A_{\alpha} \| \sum B_{\beta}$;

Now if in addition $\sum A_{\alpha}<<C$ and $\sum B_{\beta}<<D$, then (ii) and (iii) hold.

(ii) $\mathbf{E}\left(\sum A_{\alpha}\right) \| \mathbf{E}\left(\sum B_{\beta}\right)$;

(iii) $\operatorname{Hom}_{R}\left[\mathbf{E}\left(\sum B_{\beta}\right), \mathbf{E}\left(\sum A_{\alpha}\right)\right]=0$.

Proof. (i) comes from repeated application of the previous lemma. (The reason why it was not necessary to assume that $\mathbf{Z D}=0$ is that it was not necessary to assume above in 2.3 that $A+B$ was torsion free.) 
(ii) If not, then there is a submodule $0 \neq x R<C$ and an isomorphism $f: x R \longrightarrow y R \subset D$ with $f x=y$. Then $0 \neq y a=f x a \in \sum B_{\beta}$ for some $a \in R$. It now follows from $\mathbf{Z} C=0$ that for some $b \in$ $(x a)^{-1} \sum A_{\alpha}<<R, \quad b \notin(x a)^{\perp}$ and that $0 \neq x a b \in \sum A_{\alpha}$. Thus $0 \neq$ $f(x a b)=y a b$ and $\sum A_{\alpha} \supseteq x a b R \cong y a b R \subset \sum B_{\beta}$ contradicts 2.4(i).

(iii) In general, if $\mathbf{Z C}=0$, then

$$
C \| D \Longleftarrow \operatorname{Hom}_{R}(\hat{D}, \hat{C})=0
$$

2.5. Definition. Two modules $U$ and $V$ are related if first they are both uniform, and secondly, if they have isomorphic injective hulls $\hat{U}$ $\cong \hat{V}$. A type $\tau$ or $\rho$ is an equivalence class of related torsion free uniform modules. The disjoint types form a set $\Xi=\{\tau, \rho, \ldots\}$ of cardinality at most $|\Xi| \leq\left|R / \mathbf{Z}_{2} R\right|$.

Let $\Omega$ be any class of torsion free uniform modules which is closed under nonzero submodules, injective hulls, and under isomorphic copies, i.e. $\Omega$ contains all modules isomorphic to any member of itself. Note that $\Omega$ is saturated with respect to this equivalence relation, i.e. $\Omega$ is the disjoint union $\Omega=\tau \cup \rho \cup \cdots$ of types.

For a torsion free module $M$ only, define a unique submodule $M_{\Omega} \leq M$ by $M_{\Omega}=\sum\{U \mid U \leq M, U \in \Omega\}$. Two extreme cases are when $\Omega=\tau$ is a single equivalence class, and when $\Omega$ is the class $\Omega=\Sigma$ of all torsion free uniform modules. A torsion free module $M$ will be said to be of type $\tau$ provided $M=M_{\tau}$, and of generalized type $\Omega$ if $M=M_{\Omega}$.

2.6. Type decompositions. For any torsion free module $M$ and class $\Omega$ as in 2.5 ,

(i) $(\mathbf{E} M)_{\Omega} \subseteq \mathbf{E}\left(M_{\Omega}\right)$

(ii) $\mathbf{E}\left(M_{\Sigma}\right)=\mathbf{E}\left(M_{\Omega}\right) \oplus \mathbf{E}\left(M_{\Sigma \backslash \Omega}\right), \quad \mathbf{E}\left(M_{\Omega}\right) \| \mathbf{E}\left(M_{\Sigma \backslash \Omega}\right) \quad$ and $\operatorname{Hom}_{R}\left[\mathbf{E}\left(M_{\Omega}\right), \mathbf{E}\left(M_{\Sigma \backslash \Omega}\right)\right]=0$.

(iii) Any decomposition of $\Omega$ as a disjoint union $\Omega=\cup \Omega(\rho)$ of saturated subsets $\Omega(\rho)$ in the sense of 2.5 , where $\rho$ ranges over some index set, not only induces a corresponding direct sum decomposition of $M_{\Omega}$, but moreover

$$
M_{\Omega}=\oplus M_{\Omega(\rho)}, \quad \mathbf{E}\left(M_{\Omega}\right)=\mathbf{E}\left[\oplus \mathbf{E}\left(M_{\Omega(\rho)}\right)\right] .
$$

In particular,

(iv) $M_{\Omega}=\oplus\left\{M_{\rho} \mid \rho \in \Xi, \rho \cap \Omega \neq \varnothing\right\}$.

(v) $M_{\Omega}$ contains an essential direct sum of submodules from $\Omega$. 
(vi) If in addition to $\mathbf{Z} M=0, M$ is injective $M=\hat{M}$, then $M_{\Omega}$ is a direct sum of indecomposable injectives from $\Omega$. Hence in particular for $\Omega=\tau \in \Xi$, or for $\Omega=\Sigma$, the submodules $M_{\tau} \leq M_{\Sigma}$ are direct sums of indecomposable injectives.

Proof. (i) If $\xi \in(\mathbf{E} M)_{\Omega}$, then $\xi=q_{1}+\cdots+q_{n} \in Q_{1}+\cdots+Q_{n} \subseteq$ $\mathbf{E} M$ for some $0 \neq q_{i} \in Q_{i} \in \Omega$. Then $0 \neq q_{i} b_{i} \in M$ for some $b_{i} \in R$. Since $q_{i} b R \in \Omega, q_{i} b_{i} R \subseteq M_{\Omega}$. But then the latter together with the uniqueness of injective hulls inside torsion free modules implies that $q_{i} \in \mathbf{E}\left(q_{i} R\right)=\mathbf{E}\left(q_{i} b_{i} R\right) \subseteq \mathbf{E}(M)$.

(ii), (iii), and (iv): These follow from 2.4. (v): By Zorn. (vi) Conclusion (vi) follows from (v) and the type of argument in (i).

A variation of (i), (ii), and (iv) below is given in [MüR; p. 296, Theorem 1]. Much of the substance of the next theorem is in the three corollaries, and it would be interesting to see if some of 2.7-2.10 below can be extended to the generalized quasi-injective modules of Müller and Rizvi.

2.7. THEOREM I. Every injective module $M$ is of the form

$$
M=C \oplus C^{\prime} \oplus D^{\prime} \oplus D
$$

where

(i) $D \oplus D^{\prime}$ contains an essential direct sum of uniform submodules;

(ii) $C \oplus C^{\prime}$ contains no uniform submodules.

(iii) $\mathbf{Z}_{\mathbf{2}} M=C^{\prime} \oplus D^{\prime}$.

(iv) Uniqueness: If $M=C_{1} \oplus C_{1}^{\prime} \oplus D_{1}^{\prime} \oplus D_{1}$ satisfying (i), (ii) and (iii) correspondingly, then

(a) $C^{\prime} \oplus D^{\prime}=C_{1}^{\prime} \oplus D_{1}^{\prime}$ and $C^{\prime} \cong C_{1}^{\prime}, D^{\prime} \cong D_{1}^{\prime}$.

(b) $D \cong D_{1}$ and $C \cong C_{1}$.

(v) $M=\mathbf{Z}_{\mathbf{2}} M \oplus C \oplus D, \mathbf{Z}_{\mathbf{2}} M=\mathbf{E}(\mathbf{Z} M)$

(vi) $D=\mathbf{E}\left[\oplus\left\{D_{\tau} \mid \tau \in \Xi\right\}\right]$

(vii) $\operatorname{Hom}_{R}\left[\mathbf{E}\left(D_{\tau}\right), \mathbf{E}\left(D_{\rho}\right)\right]=0$ for $\tau \neq \rho$.

Proof. (i) and (ii). Let $M=F \oplus G$ as in 2.1. Since for any module $W$, $\mathbf{Z}_{\mathbf{2}} W<W$ is a complement submodule, write $F=C \oplus C^{\prime}$ and $G=$ $D \oplus D^{\prime}$ where $C^{\prime}=\mathbf{Z}_{2} F$ and $D^{\prime}=\mathbf{Z}_{2} G$, and $C$ and $D$ are some complementary direct summands.

(iii) After verifying that $\mathbf{Z}_{\mathbf{2}}$ is an additive functor, we conclude that $\mathbf{Z}_{\mathbf{2}} M=\mathbf{Z}_{\mathbf{2}}(F \oplus G)=C^{\prime} \oplus D^{\prime}$. 
(iv) By 2.1, $C \oplus C^{\prime} \cong C_{1} \oplus C_{1}^{\prime}$ and $D \oplus D^{\prime} \cong D_{1} \oplus D_{1}^{\prime}$. It follows from (iii) that $\mathbf{Z}_{2} M=C^{\prime} \oplus D^{\prime}=C_{1}^{\prime} \oplus D_{1}^{\prime}$.

(a) In Lemma 2.1 , replacing $M$ by $\mathbf{Z}_{2} M$ which by (iii) is $\mathbf{Z}_{2} M=$ $C^{\prime} \oplus D^{\prime}=C_{1}^{\prime} \oplus D_{1}^{\prime}$, we conclude that $C^{\prime} \cong C_{1}^{\prime}$ and $D^{\prime} \cong D_{1}^{\prime}$.

(b) Since $M=\mathbf{Z}_{2} M \oplus C \oplus D=\mathbf{Z}_{2} M \oplus C_{1} \oplus D_{1}$, it follows that $C \oplus D \cong C_{1} \oplus D_{1}$. Again 2.1 implies that $C \cong C_{1}$ and $D \cong D_{1}$.

(v) Since $\mathbf{E Z}_{\mathbf{2}} M=\mathbf{Z}_{\mathbf{2}} \mathbf{E} M$, and $\mathbf{Z} M<<\mathbf{Z}_{\mathbf{2}} M, \mathbf{E}(\mathbf{Z} M)=\mathbf{Z}_{\mathbf{2}} M$.

(vi) By 2.6 (iv), $D_{\Sigma}=\oplus\left\{D_{\tau} \mid \tau \in \Xi\right\}<<D$.

(vii) With $M=D$ and $\Omega=\tau, 2.6\left(\right.$ ii) shows that $\operatorname{Hom}_{R}\left[\mathbf{E}\left(D_{\tau}\right), \mathbf{E}\left(D_{\rho}\right)\right]$ $\subseteq \operatorname{Hom}_{R}\left[\mathbf{E}\left(D_{\tau}\right), \mathbf{E}\left(D_{\Sigma \backslash \tau}\right)\right]=0$.

2.8. Corollary 1 to Theorem I. If $M$ is any torsion free injective module and if $M=C \oplus D=C_{1} \oplus D_{1}$ are two decompositions into continuous and discrete submodules as given by the last theorem, then

(i) $C$ and $D$ are fully invariant submodules of $M$.

(ii) Uniqueness: $D=D_{1}$ and $C=C_{1}$.

(iii) $D$ is the unique smallest injective submodule of $M$ which contains every uniform submodule of $M$.

(iv) $C$ is the unique smallest injective submodule of $M$ which contains every continuous submodule of $M$.

Proof. (i) Define $j_{C}: C \longrightarrow M, \pi_{C}: M \longrightarrow C, j_{D}$, and $\pi_{D}$ to be the natural inclusions and projections. Take $\phi \in$ End $_{R} M$ arbitrary. The kernel of any homomorphism mapping an injective module into a torsion free module is a direct summand. Hence $D=A \oplus \operatorname{ker}\left(\pi_{C} \phi j_{D}\right)$ and $C=B \oplus \operatorname{ker}\left(\pi_{D} \phi j_{C}\right)$ for some $A<D$ and $B<C$. Then $B \cong \pi_{D} \phi B<D$ is a uniform free submodule of $D$, and hence $B=0$. Thus $\pi_{D} \phi C=0$, and $\phi C \subseteq C$. Similarly, also $\phi D \subseteq D$.

(ii) Since $C$ is fully invariant in $M, C=\left(C \cap C_{1}\right) \oplus\left(C \cap D_{1}\right)=$ $C \cap C_{1}$, and similarly $D=D \cap D_{1}$. Thus $C=C_{1}$ and $D=D_{1}$.

(iii) If $x R<M$ is uniform, then since $D$ contains a maximal essential direct sum of uniform submodules, necessarily $0 \neq x b \in D$ for some $b \in R$. Since $\mathbf{Z} M=0, x \in \mathbf{E}(x R)=\mathbf{E}(x b R) \subseteq D$.

(iv) If $B<M$ is any continuous submodule, then a decomposition $M=C_{1} \oplus D_{1}$ in 2.1 may be so chosen that $\hat{B} \subset C_{1}=C$.

2.9. Corollary 2 to Theorem I. As in the last corollary, let $M=$ $C \oplus D$ be the decomposition of a torsion free injective module $M$ and $\tau \in \Xi$ any equivalence class. Then

(i) $\oplus_{\tau \in \Xi} \mathbf{E}\left(D_{\tau}\right)<<D=\Pi_{\tau \in \Xi} \mathbf{E}\left(D_{\tau}\right)$. 
(ii) Uniqueness: $D_{\tau}, \mathbf{E}\left(D_{\tau}\right), \oplus \mathbf{E}\left(D_{\tau}\right)$, and $D$ are intrinsic fully invariant submodules of $M$.

Proof. (i) Firstly, $D_{\Sigma}<<D$ by definition of $D$; secondly, 2.6 (iv) shows that $D_{\Sigma}=\oplus D_{\tau}$, and hence thirdly, that $\oplus \mathbf{E}\left(D_{\tau}\right)<<D \subseteq$ $\Pi \mathbf{E}\left(D_{\tau}\right)$. It suffices to show that if $0 \neq \xi=\left(\xi_{\tau}\right) \in \Pi \mathbf{E}\left(D_{\tau}\right)$ with $0 \neq \xi_{\tau} \in$ $\mathbf{E}\left(D_{\tau}\right)$ is arbitrary, that then there exists an $r \in R$ such that $0 \neq \xi r \in$ $\mathbf{E}\left(D_{\tau}\right) \subset \Pi \mathbf{E}\left(D_{\tau}\right)$. (Note that at this point we may not assume that $\xi \in M$.) Since $\xi_{\tau}^{\perp}<\nless R$, take any $0 \neq V_{\tau}<R$ with $\xi_{\tau}^{\perp} \cap V_{\tau}=0$. Suppose that $\xi_{\rho} V_{\tau} \neq 0$ for some $\tau \neq \rho \in \Sigma$. Since $\xi_{\rho} \in \mathbf{E}\left(D_{\rho}\right), 0 \neq \xi_{\rho} V_{\tau}<$ $\mathbf{E}\left(D_{\rho}\right)$. Form the commutative diagram

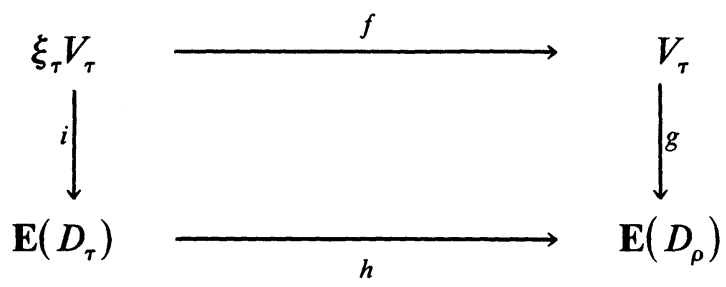

where $i$ is the natural inclusion, $g$ is left multiplication by $\xi_{\rho}$, that is $g v=\xi_{\rho} v, v \in V_{\tau} ; f$ is the isomorphism $f\left(\xi_{\tau} v\right)=v$, while lastly, by the injectivity of $\mathbf{E}\left(D_{\rho}\right)$, hi $=g f$. Then $g f\left(\xi_{\tau} V_{\tau}\right)=g\left(V_{\tau}\right)=\xi_{\rho} V_{\tau} \neq 0$. Hence $0 \neq h \in \operatorname{Hom}_{R}\left[\mathbf{E}\left(D_{\tau}\right), \mathbf{E}\left(D_{\rho}\right)\right]$ contradicts 2.7(vii). Thus $V_{\tau} \subset \bigcap\left\{\xi_{\rho}^{\perp} \mid \tau \neq\right.$ $\rho \in \Xi\}$, and consequently for any $0 \neq r \in V_{\tau}, 0 \neq \xi r=\xi_{\tau} r \in \mathbf{E}\left(D_{\tau}\right)$. Thus $D=\Pi \mathbf{E}\left(D_{\tau}\right)$.

(ii) For a torsion free injective module $M, M_{\tau}=(C \oplus D)_{\tau}=D_{\tau}$, and $M_{\Sigma}=\oplus D_{\tau}$ are intrinsically defined. Let $\phi \in$ End $_{R} M$ be arbitrary. From $D_{\tau}<<\mathbf{E}\left(D_{\tau}\right)$ together with $\mathbf{Z} M=0$ we can conclude that also $\phi D_{\tau}<<\phi \mathbf{E}\left(D_{\tau}\right)$. But $\phi D_{\tau} \subseteq D_{\tau}$, and hence $\phi\left(\mathbf{E}\left(D_{\tau}\right)\right) \subseteq \mathbf{E}\left(D_{\tau}\right)$ is fully invariant.

2.10. Corollary 3 to Theorem I. Let $R=C \oplus D$ be any decomposition as in Theorem I of a torsion free right self injective ring $R$. Then for any types $\rho \neq \tau \in \Xi$, the following hold.

(i) $C \triangleleft R, D \triangleleft R ; C D=D C=0$.

(ii) $C, D \triangleleft R, D_{\tau} \triangleleft R$, and $\mathbf{E}\left(D_{\tau}\right) \triangleleft R$ are intrinsic ideals which are fully invariant as right $R$-submodules of $R$. 0.

(iii) $D=\mathbf{E}\left(\oplus D_{\tau}\right)=\Pi \mathbf{E}\left(D_{\tau}\right)$ as rings. In particular, $\mathbf{E}\left(D_{\rho}\right) \mathbf{E}\left(D_{\tau}\right)=$

(iv) Each $\mathbf{E}\left(D_{\tau}\right)$ is a right full linear ring. 
(v) If the hypothesis that $R$ is right self injective is omitted, then (i)-(iv) are satisfied by the ring $\hat{R}=C \oplus D$, if every occurrence of $R$ above is replaced by $\hat{R}$.

Proof. (i) and (ii). Fully invariant right ideals are two sided. Note that $\mathbf{E}\left(D_{\rho}\right) \mathbf{E}\left(D_{\tau}\right)=0$ is a simple consequence of the fact that $\mathbf{E}\left(D_{\rho}\right) \oplus \mathbf{E}\left(D_{\tau}\right) \triangleleft R$.

(iii) By 2.9(i), $D=\mathbf{E}\left(\oplus D_{\tau}\right)=\Pi \mathbf{E}\left(D_{\tau}\right)$ as right $R$-modules. Furthermore the product carries two ring structures: The one inherited from the subring $D$, as well as its own independent infinite product ring structure (since by (ii) the $\mathbf{E}\left(D_{\tau}\right)$ are rings). By (i) these two ring structures carry over to $R=C \oplus D=C \oplus \Pi \mathbf{E}\left(D_{\tau}\right)$. Let $\mathbb{Z}=0, \pm 1, \pm 2, \ldots$ and $1_{R} \in R$ be the identity. These two ring structures on $R$ coincide on $C \oplus$ $\left\{\oplus \mathbf{E}\left(D_{\tau}\right)\right\}+\mathbb{Z} 1_{R}$. At this point we use the following well known fact. The ring structure of a torsion free ring with identity like $C \oplus\left\{\oplus \mathbf{E}\left(D_{\tau}\right)\right\}$ $+\mathbb{Z} 1_{R}$ has a unique extensions to its right $R$-injective hull $\hat{R}$ which is compatible with the right $R$-submodule structure of this ring as a submodule of $\hat{R}$. Conclusion (iv) is known and (v) the passage from $R$ to $\hat{R}$ when $\mathbf{Z} R=0$ is standard.

From now on till the end of this section $M$ is an arbitrary unital right $R$-module (with $\mathbf{Z} M \neq 0$ allowed).

2.11. Definition. Let $\Omega$ be any saturated class of torsion free uniform modules (as in 2.5). The $\Omega$-dimension of any module whatever-denoted by $\Omega-d M-$ is $\Omega-d M=|J|$, where $\oplus\left\{U_{j} \mid j \in J\right\}$ $\leq M$ is any direct sum of submodules $U_{j} \in \Omega$ maximal in $M$ with respect to $\Omega$ in the sense that for any uniform $V \leq M$, if $V \in \Omega$, then necessarily $V \cap \oplus U_{j} \neq 0$.

2.12. Methods of computing $\Omega-d M$. For $\Omega \subseteq \Sigma$, and $M$ as above, let $\oplus\left\{U_{i} \mid i \in I\right\} \leq M$ be any direct sum of torsion free uniform submodules $U_{i}<M$ whatever, maximal with respect to $\Sigma$. Define a subset $J \subseteq I$ as $J=\left\{i \in I \mid U_{i} \in \Omega\right\}$. Use a routine step by step Zorn's lemma argument to decompose $\hat{M}=\mathbf{Z}_{2} \hat{M} \oplus C \oplus D$ as in Theorem I with $\oplus U_{i}<<D$. Then

(1) $\Omega-d M$ is well defined.

(2) (i) $\Sigma-d M=|I|$,

(ii) $\Omega-d M=|J|$. 
Proof. (1) and (2). Let $\oplus\left\{V_{\beta} \mid \beta \in \mathfrak{B}\right\} \leq M$ be any second direct sum, with all $V_{\beta} \in \Omega$, and maximal in $M$ with respect to $\Omega$ as in 2.11. Extend this family $\left\{V_{\beta} \mid \beta \in \mathfrak{U}\right\} \subseteq\left\{V_{\alpha} \mid \alpha \in \mathfrak{U}\right\}$ so that $\oplus\left\{V_{\alpha} \mid \alpha \in \mathfrak{A}\right\}$ $\leq M$ is a maximal direct sum of torsion free uniform submodules of $M$. Write $\hat{M}=\mathbf{Z}_{2} \hat{M} \oplus C \oplus D=\mathbf{Z}_{2} \hat{M} \oplus C_{1} \oplus D_{1}$ as in Theorem I with $\oplus V_{\alpha}<<D_{1}$, where $D=\mathbf{E}\left(\oplus\left\{U_{i} \mid i \in I\right\}\right) \cong D_{1}=\mathbf{E}\left(\oplus\left\{V_{\alpha} \mid \alpha \in \mathfrak{A}\right\}\right)$. By [W; p. 271, Corollary 4.2], there is a bijection $\pi: I \longrightarrow$ such that $\hat{U}_{i} \cong \hat{V}_{\pi i}$ for all $i \in I$. Consequently, $\pi(J)=\mathfrak{A}$. Thus $\Sigma-d M=$ $|I|=|\mathfrak{A}|$ and $\Omega-d M=|J|=|\mathfrak{B}|$ are well defined.

2.13. Proposition. For any saturated class $\Omega \subseteq \Sigma$ and with $M=R$, use the construction in 2.12 (or any other method) to obtain $\mathbf{Z}_{\mathbf{2}} R \oplus F \oplus G$ $\oplus H<<R$, where $F$ contains no uniform right ideals, whereas $G$ and $H$ are direct sums of uniform right ideals $U_{i}$, where $G$ is maximal with respect to $\Sigma \backslash \Omega$ in $R$ and $H$ with respect to $\Omega$ as in 2.11 .

$$
\begin{aligned}
& G=\bigoplus_{i \in \Lambda J} U_{i}, \quad\left\{U_{i} \mid i \in I \backslash J\right\} \subset \Sigma \backslash \Omega, \quad J \subset I, \\
& H=\bigoplus_{i \in J} U_{j}, \quad\left\{U_{j} \mid j \in J\right\} \subset \Omega .
\end{aligned}
$$

Now let $K \leq R$ be (the unique) complement right ideal such that $\mathbf{Z}_{2} R \oplus F$ $\oplus G<<K$, and hence $K \oplus H<<R$. Then for every $W \in \Omega, K \subset W^{\perp}$. In particular, $H K=0$.

Proof. Let $0 \neq y \in W$. Any $g \in G$ is of the form $g=g_{1}+\cdots+g_{n}$, where $g_{i} R \in \Sigma \backslash \Omega$ are uniform. Since $\mathbf{Z}\left(g_{l} R\right)=0$, necessarily $y g_{i} R \neq 0$, because otherwise $0 \neq y g_{i} R \subset W \in \Omega$ and $y g_{i} R \cong g_{i} R \in \Omega \cap(\Sigma \backslash \Omega)=$ $\varnothing$ is a contradiction. Hence $y g R=0, y G=0$.

If $y F \neq 0$, there exists an $f \in F$ such that $0 \neq y f R \in \Omega$. But $y f R \cong$ $f R /\left(y^{\perp} \cap f R\right)$. Since $f R$ is uniform free while $f R /\left(y^{\perp} \cap f R\right)$ is uniform, $y^{\perp} \cap f R<<f R$. Then $f R /\left(y^{\perp} \cap f R\right)$ is torsion, a contradiction. Hence $y F=0$.

Thus far $\mathbf{Z}_{\mathbf{2}} R \oplus F \oplus G \subseteq W^{\perp}$. Since $\mathbf{Z}_{\mathbf{2}} R \oplus F \oplus G<<K$, for any $k \in K, k^{-1}\left(\mathbf{Z}_{\mathbf{2}} R \oplus F \oplus G\right)<<R$. Thus $(y k)\left[k^{-1}\left(\mathbf{Z}_{\mathbf{2}} R \oplus F \oplus G\right)\right]=0$ implies that $y k \in \mathbf{Z} W=0$. Hence $K \subseteq W^{\perp}$.

3. Injectivity of $\sigma$-products. Starting with a fixed infinite cardinal $\sigma$ and some class of modules - such as torsion free, or torsion free uniform, or torsion free uniform of type $\Omega$-necessary and sufficient conditions are 
found in order for every $\sigma$-product of injective modules of the respective class to be injective.

3.1. Dimension Theorem II. For a module $M$, suppose that $A_{i}<M$, $i \in I$, is a family of submodules of $M$ whose (not necessarily direct) sum $\sum\left\{A_{i} \mid i \in I\right\}<<M$ is large in $M$. Then there exists a single index $m \in I$ such that already $A_{m}<<M$ is large provided that we can find an infinite cardinal $\sigma$ and a complement submodule $K<M$ satisfying the following conditions.

(a) $\mathbf{Z} M \subseteq K$, and $K \subseteq A_{i}$ for all $i$.

(b) Every nontrivial direct sum of submodules of $M / K$ contains strictly less than $\sigma$ nonzero direct summands.

(c) For any $J \subseteq I$, if $|J|<\sigma$, then there exists a $k \in I$ such that $\sum\left\{A_{j} \mid j \in J\right\} \subseteq A_{k}$.

Proof. Let $\mathscr{S}$ be the non-empty set consisting of all pairs $\left(J,\left\{A_{J}^{J} \mid\right.\right.$ $j \in J\}$ ), where $\varnothing \neq J \subseteq I$, and where $0 \neq A_{j}^{J} \leq A_{j}$ for all $j \in J$, such that, furthermore, the sum

$$
K+\sum A_{j}^{J}=K \oplus\left[\oplus\left\{A_{j}^{J} \mid j \in J\right\}\right] \leq M
$$

is direct. The set $\mathscr{S}$ is partially ordered by

$$
\left(N,\left\{A_{j}^{N}\right\}\right) \leq\left(J,\left\{A_{j}^{J}\right\}\right) \Longleftrightarrow \begin{aligned}
& N \subseteq J \text { and } \\
& A_{j}^{N} \subseteq A_{n}^{J} \text { for all } n \in N .
\end{aligned}
$$

By Zorn, the inductively ordered set $\mathscr{S}$ contains a maximal element $\left(J,\left\{V_{j} \mid j \in J\right\}\right)$.

If $K \oplus\left[\oplus V_{j}\right]<<M$, hypothesis (b) shows that $|J|<\sigma$. Then by (c), there exists an $m \in I$ with $\sum\left\{A_{j} \mid j \in J\right\} \subseteq A_{m}$. But since $K+V_{j} \subseteq A_{j}$, it now follows that $K+\sum V_{j} \subseteq A_{m}<<R$ as required.

In the remaining other case when $K \oplus\left[\oplus V_{j}\right]<\nless M$ is not essential, since $\sum A_{i}<<M$, there eixsts a direct sum

$$
\begin{gathered}
K \oplus\left[\oplus V_{j}\right] \oplus\left(a_{i(1)}+\cdots+a_{i(n)}\right) R, \\
0 \neq a_{i(k)} \in A_{i(k)} \backslash\left(K \oplus\left[\oplus V_{j}\right]\right), \quad k=1, \ldots, n ;
\end{gathered}
$$

where $a_{i(1)}+\cdots+a_{i(n)} \neq 0$, for some $i(1), \ldots, i(n) \in I$. Next, it will be shown that each of the right ideals $L_{k}=a_{i(k)}^{-1}\left(K \oplus\left[\oplus V_{j}\right]\right)$ is large. For if not, then $L_{k} \oplus B \leq R$ for some $0 \neq B \leq R$. Then $K \oplus\left[\oplus V_{j}\right] \oplus a_{i(k)} B$ is a direct sum with $0 \neq a_{i(k)} B \cong B$. If $i(k) \notin J$, then $\left(J \cup\{i(k)\},\left\{V_{j} \mid\right.\right.$ $\left.j \in J\} \cup\left\{a_{i(k)} B\right\}\right) \in \mathscr{S}$ is a contradiction. If, however, $i(k) \in J$, then $V_{i(k)} \lesseqgtr V_{l(k)}+a_{i(k)} B \subseteq A_{i(k)}$ again contradicts maximality. Therefore $L_{k}$ 
is large, and so also is $\bigcap_{1}^{n} L_{k}<<R$. Thus

$$
\begin{aligned}
& \left(a_{i(1)}+\cdots+a_{i(n)}\right) R\left[\cap_{1}^{n} L_{k}\right] \\
& \quad \subseteq\left(K+\sum V_{j}\right) \cap\left(a_{i(1)}+\cdots+a_{i(n)}\right) R=(0),
\end{aligned}
$$

and hence $a_{i(1)}+\cdots+a_{i(n)} \in \mathbf{Z} M \subseteq K$ is a contradiction.

Although the author does not know how much if any of the theory can be modified to cover the torsion or mixed case, the next proposition might well be the first step in this direction. It omits the hypothesis that $\mathbf{Z} M \subseteq K$, and hence the previous method of proof fails. However, severe restrictive hypotheses on $M / K$ make the next proposition much easier to prove than the last theorem. Furthermore, the next corollary is all that is required later to prove Theorem IV.

3.2. Proposition. Suppose that $A_{i}<M, i \in I$, with $\sum A_{i}<<M$. Then $A_{m}<<M$ for some $m \in I$ if for some infinite cardinal $\sigma$ and complement $K<M$ the following hold.

(a) $K \subseteq A_{i}$ for all $i$.

(b) $M / K$ contains an essential direct sum of uniform submodules containing strictly less than $\sigma$ summands.

(c) Same as in 3.1.

Proof. Let $K \oplus\left[\oplus\left\{U_{\lambda} \mid \lambda \in \Lambda\right\}\right]<<M$ where the $U_{\lambda}$ are uniform. Since $\sum A_{i}<<M$, for each $\lambda \in \Lambda$, there exists $0 \neq x_{\lambda} \in U_{\lambda} \cap \sum A_{i}$. By (c), $\sum A_{i}=\bigcup A_{i}$, and as a consequence we can select $j(\lambda) \in I$ such that $x_{\lambda} \in A_{j(\lambda)}$. Set $J=\{j(\lambda) \mid \lambda \in \Lambda\}$ by [W; p. 271, Corollary 4.2], $|J|=$ $|\Lambda|<\sigma$. Hence by (c) there exists an $m$ and an $A_{m}$ such that $\sum A_{j(\lambda)} \subseteq A_{m}$. It follows from (a) that $K \oplus\left[\oplus\left\{x_{\lambda} R \mid \lambda \in \Lambda\right\}\right] \subseteq A_{m}$. However, $K \oplus\left[\oplus x_{\lambda} R\right]<<R$. Thus also $A_{m}<<R$.

In (1) below, since $\mathbf{Z}_{\mathbf{2}} R \triangleleft R$, the right $R$-submodules of the $R$-module $R / \mathbf{Z}_{2} R$ are precisely the right ideals of the ring $R / \mathbf{Z}_{2} R$.

3.3. General THeOrem III. Let $R$ be a ring with an identity element, $\mathbf{Z}_{2} R<R$ the second right singular submodule, and $\sigma$ a regular cardinal. Then the following three conditions (1), (2), and (3) are all equivalent.

(1) Every direct sum of right $R$-submodules of $R / \mathbf{Z}_{\mathbf{2}} R$ contains strictly less than $\sigma$ nonzero summands.

(2) Every $\sigma$-product of torsion free injective right $R$-modules is injective.

(3) Every $\sigma$-product of torsion free right $R$-modules is a complement submodule of their full direct product. 
Proof. It will be shown that (1) $\Longrightarrow$ (3) $\Longrightarrow(2) \Longrightarrow$ (1), and that only the first of these implications requires $\sigma$ to be regular.

(1) $\Longrightarrow$ (3). Let $\left\{W_{\gamma} \mid \gamma \in \Gamma\right\}$ be any indexed collection of torsion free modules $W_{\gamma}$. It suffices to show that for any $x=\left(x_{\gamma}\right) \in \Pi W_{\gamma}$ such that $\Pi^{\sigma} W_{\gamma}<<\Pi^{\sigma} W_{\gamma}+x R$, necessarily also $x \in \Pi^{\sigma} W_{\gamma}$. Define $I=$ $\{i \subset \Gamma|| i \mid<\sigma\}$ and $A_{i}=\bigcap\left\{x_{\gamma}^{\perp} \mid \gamma \notin i\right\}$. This implies that $x^{-1} \Pi^{\sigma} W_{\gamma}=$ $\cup\left\{A_{i} \mid i \in I\right\}<<R$ (see 1.6 and 1.2).

First, assume that $A_{m}<<R$ for some $m \in I$ where $m \subset \Gamma$ with $|m|<\sigma$. For every $\beta \notin m$, we have $A_{m} \subset x_{\beta}^{+}$, and hence also that $x_{\beta}^{\perp}<<R$. Then $x_{\beta} \in \mathbf{Z} W_{\beta}=0$, consequently $\operatorname{supp} x \subseteq m$, and finally $x \in \Pi^{\sigma} W_{\gamma}$. Thus it has been shown that $\Pi^{\sigma} W_{\gamma}<\Pi W_{\gamma}$ is a complement provided that some $A_{m}<R$.

It will now be shown that $I$ and the $A_{i}$ satisfy conditions (a), (b), and (c) of 3.1 with $K=\mathbf{Z}_{2} R<R=M$, which is a complement (see 1.2(f)). For every $\gamma, W_{\gamma}\left(\mathbf{Z}_{2} R\right)=0$, and thus (a) $\mathbf{Z}_{2} R \subseteq A_{i}$ holds for all $i$, while (b) holds by the hypothesis (1).

In order to establish the remaining condition (c), suppopse that $J \subseteq I$ with $|J|<\sigma$. Next, define $k=\bigcup J=\bigcup\{j \mid j \in J\} \subset \Gamma$. Each member $j \in J$ satisfies $|j|<\sigma$, and the total number $|J|$ of such $j$ appearing in the union for $k$ also satisfies $|J|<\sigma$. But then likewise

$$
|k|=\sum_{j \in J}|j|<\sigma
$$

because $\sigma$ is regular. Since $j \subset k$ for all $j \in J$, also $A_{j} \subseteq A_{k}$. Thus the condition (c) that $\Sigma\left\{A_{j} \mid j \in J\right\} \subseteq A_{k}$ also holds. Hence by the Dimension Theorem, there exists an $m \in I$ such that $A_{m}<<R$.

(3) $\Longrightarrow(2)$ is trivial.

(2) $\Longrightarrow$ (1). Since $\mathbf{Z}_{2} R<R$ is a complement, any nontrivial direct sum in $R / \mathbf{Z}_{2} R$ indexed by a set $\Lambda$, lifts to a corresponding nontrivial direct sum of right ideals $\mathbf{Z}_{\mathbf{2}} R \oplus\left[\oplus\left\{V_{\lambda} \mid \lambda \in \Lambda\right\}\right] \leq R$. Hence it suffices to show that $|\Lambda|<\sigma$. By hypothesis (2), the identity, that is the inclusion map $\phi: \oplus V_{\lambda} \longrightarrow \Pi^{\sigma} \hat{V}_{\lambda}$ extends to $\hat{\phi}: R \longrightarrow \Pi^{\sigma} \hat{V}_{\lambda}$. For any $\lambda \in \Lambda$, and any $0 \neq r \in V_{\lambda} \subset R,(\hat{\phi} 1) r=\phi r=r \in V_{\lambda} \subset \Pi^{\sigma} V_{\lambda}$ shows that $\lambda \in \operatorname{supp} \hat{\phi} 1$. Consequently supp $\hat{\phi} 1=\Lambda$, and $|\Lambda|=|\operatorname{supp} \hat{\phi} 1|<\sigma$.

3.4. COROLlARY TO THEOREM III. In general, for every ring $R$, there exists a unique smallest infinite regular cardinal $\sigma(R) \leq\left|R / \mathbf{Z}_{\mathbf{2}} R\right|^{+}$such that $R$ satisfies conditions (1)-(3) of the previous theorem for all regular cardinals $\sigma \geq \sigma(R)$. (See 1.3.) 
3.5. Theorem IV. For a ring $R$, suppose that $\Omega$ is any class of torsion free uniform modules closed with respect to containing nonzero submodules and injective hulls of all of its members, and that $\Omega-d R$ is the $\Omega$-dimension of $R$. Then for any regular cardinal $\sigma$, the following are all equivalent.

(1) $\Omega-d R<\sigma$.

(2) For any family of torsion free indecomposable injective modules $\hat{W}_{\gamma} \in \Omega, \gamma \in \Gamma$, for arbitrary $\Gamma$, their o-product $\Pi^{\sigma}\left\{\hat{W}_{\gamma} \mid \gamma \in \Gamma\right\}$ is injective.

(3) For any torsion free uniform modules $W_{\gamma} \in \Omega, \gamma \in \Gamma$;

$$
\Pi^{\sigma}\left\{W_{\gamma} \mid \gamma \in \Gamma\right\}<\Pi\left\{W_{\gamma} \mid \gamma \in \Gamma\right\}
$$

is a complement submodule.

3.6. Corollary to Theorems III AND IV. In both the General Theorem III as well as in the last Theorem IV, the implications (3) $\Longrightarrow$ $(2) \Longrightarrow$ (1) always hold for any cardinal $\sigma$, both regular or singular.

Proof of 3.5 and 3.6. (3) $\Longrightarrow$ (2) is trivial. (2) $\Longrightarrow$ (1). Suppose that $\mathbf{Z}_{2} R \oplus\left[\oplus\left\{U_{\lambda} \mid \lambda \in \Omega\right\}\right] \leq R$ is a maximal direct sum of $U_{\lambda} \in \Omega$ as in 2.11. Extend the inclusion map $\phi: \oplus U_{\lambda} \longrightarrow \Pi^{\sigma} \hat{U}_{\lambda}$ to $\hat{\phi}: R \longrightarrow \Pi^{\sigma} \hat{U}_{\lambda}$. Then $\Omega-d R=|\Lambda|=|\operatorname{supp} \hat{\phi} 1|<\sigma$.

$(1) \Longrightarrow(3)$. It suffices to show that for any $x=\left(x_{\gamma}\right) \in \Pi W_{\gamma}$ and $x^{-1} \Pi^{\sigma} W_{\gamma}=\bigcup\left\{A_{l} \mid i \in I\right\}$ as in "3.3. (1) $\Longrightarrow$ (3)", the $A_{l}<R$ satisfy conditions (a), (b), and (c) of 3.2.

(a) Let $\mathbf{Z}_{\mathbf{2}} R \oplus F \oplus G \oplus H<<R$ be as in 2.13. Hence the complement $K \leq R$ produced in 2.13 satisfies $\mathbf{Z}_{\mathbf{2}} R \oplus F \oplus G<<K \subseteq K \oplus H$ $<R$ with $K \subseteq W^{\perp}$ for any $W \in \Omega$. In particular, for any $x_{\gamma} \in W_{\gamma} \in \Omega$, $K \subseteq x_{\gamma}{ }_{\gamma}$, and hence $K \subseteq A_{i}$ for all $i \in I$.

(b) Since $K<R$ is a complement, the quotient submodule $(K \oplus H) / K<<R / K$ remains essential, and $(K \oplus H) / K$ is a direct sum of uniform modules. Note that

$$
\Omega-d R=\Omega-d H=\Sigma-d H=\Sigma-d(R / K)<\sigma,
$$

where the very last inequality is $3.2(\mathrm{~b})$. Condition (c) holds as in 3.3 because $\sigma$ is regular.

3.7. ThEOREM V. Assume throughout in every one of the five condition below that

(i) every nonzero torsion free module contains a uniform submodule. 
Then for a regular cardinal $\sigma$ the following five conditions $(1),(2 *),(2)$, $\left(3^{*}\right)$, and (3) are all equivalent.

(1) (i) $R / \mathbf{Z}_{2} R$ contains an essential direct sum of uniform $R$-submodules with

(ii) strictly less than $\sigma$-summands.

(2*) Any $\sigma$-product of indecomposable injective torsion free modules is injective.

(2) Every $\sigma$-product of torsion free injective modules is injective.

$\left(3^{*}\right)$ Any $\sigma$-product of torsion free uniform modules is a complement in their direct product.

(3) Every $\sigma$-product of torsion free modules is a complement in their full direct product.

Furthermore, the implications

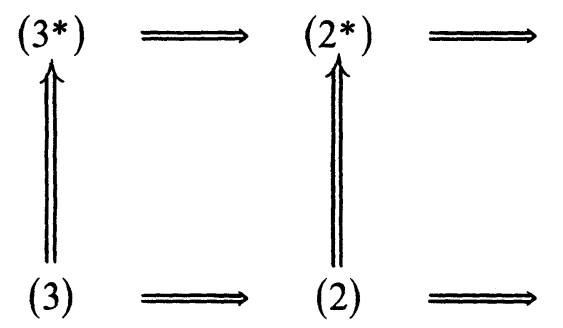

hold for any $\sigma$, regular or singular.

Proof. First, irrespective of $\sigma$, the blanket hypothesis (i) is equivalent to the condition (1)(i) by itself. Secondly, the present (1)(i) and (ii) imply the analogous condition (1) in Theorem III; and thirdly, (1)(i) and (ii) imply (1) also in the previous Theorem IV with $\Omega=\Sigma$. Hence immediately Theorem III tells us that (1)(i), (ii) while from Theorem IV we get that (1)(i), (ii) $\left(3^{*}\right)$. Trivially, $(2) \Longrightarrow\left(2^{*}\right)$ and $(3) \Longrightarrow\left(3^{*}\right)$.

3.8. RemarKs. (1) For any ring $R$ whatever, $(\Omega-d R)^{+}$is the unique smallest infinite regular cardinal such that $R$ satisfies the properties (1)-(3) in Theorem IV for all regular cardinals $\sigma \geq(\Omega-d R)^{+}$. (See 1.3.)

(2) Any ring $R$ such that $R / \mathbf{Z}_{2} R$ contains an essential direct sum of uniform submodules satisfies the following:

(a) $\sigma(R)=(\Sigma-d R)^{+}$where

$$
\Sigma-d R=\sum_{\tau \in \Xi} \tau-d R=\max (|\Xi|, \underset{\tau \in \Xi}{\operatorname{supremum}} \tau-d R)
$$

(see 3.4., 2.11, and [Le; p. 103, Proposition 4.4] or [HJ; p. 156, 2.3 and p. $152,1.2]$.) 
(b) If either $\Omega-d R<|\Xi|$, or if there exists a $\tau \in \Xi$ such that $\Omega-d R$ $<\tau-d R$, then for $\sigma=(\Omega-d R)^{+}$, the ring $R$ satisfies (1)-(3) in Theorem IV, but does not satisfy any of the conditions in Theorem III or V.

A result of M. Teply ([T2; p. 451, Theorem 2.1] and [T1; p. 442, Theorem 1.2]) is an almost immediate consequence of the last theorem with $\sigma=\boldsymbol{\aleph}_{0}$. In the special finite case when $\boldsymbol{\sigma}=\boldsymbol{\aleph}_{0}$, the blanket hypothesis (i) can be deleted from the last theorem below in Teply's Theorem, it holds automatically. Counterexamples (4.3(a) and (b)) will show that the condition (i) cannot be deleted from the last theorem.

3.9. Teply Theorem. For $\mathbf{Z}_{\mathbf{2}} R \triangleleft R$ as before, the following three conditions are equivalent.

(1) $R / \mathbf{Z}_{2} R$ contains an essential direct sum of a finite number of uniform right $R$-modules.

(2) Every direct sum of torsion free injective modules is injective.

(3) Every torsion free injective module is a direct sum of indecomposable injectives.

4. Examples and applications. The next example illustrates that if $M$ is not torsion free, that then the submodule $D$ in the decomposition $\hat{M}=\mathbf{Z}_{\mathbf{2}} \hat{M} \oplus C \oplus D$ is not unique, except up to isomorphism.

4.1. EXAmple. Set $R=\mathbb{Z}=0, \pm 1, \pm 2, \ldots$ and $M=(\mathbb{Z} / 3 \mathbb{Z}) \oplus \mathbb{Z}$. Let $D$ and $D_{1}$ be the injective hulls and $(\overline{0}, 1) \mathbb{Z}$ and $(\overline{1}, 1) \mathbb{Z}$ respectively. Then $\hat{M}=\mathbb{Z}\left(3^{\infty}\right) \oplus D=\mathbb{Z}\left(3^{\infty}\right) \oplus D_{1}$, where $D$ and $D_{1}$ are isomorphic to the rationals.

First a simple example is given which can be treated by Theorem V but not by any previously known theorems. It also illustrates that Theorem III and V cannot be formulated in terms of the more familiar $\mathbf{Z} R$, but that $\mathbf{Z}_{\mathbf{2}} R$ must be used.

4.2. EXAMPLE. With $\mathbb{Z}=0,1,2, \ldots$ set $Z_{4}=\mathbb{Z} / 4 \mathbb{Z}$, and let $e_{i j}$ denote the usual $2 \times 2$ matrix units. Define $R$ to be the upper triangular matrix ring $R=Z_{4} e_{11}+\oplus_{1}^{\infty} Z_{4} e_{12}+\Pi_{1}^{\infty} \mathbb{Z} e_{22}$, where the index set is the natural integers, the product is a ring under componentwise operations, and where the product acts on the direct sum by corresponding component acting on corresponding component: $x=\left(x_{n}\right) \in \oplus_{1}^{\infty} Z_{4}, r=\left(r_{n}\right) \in \Pi_{1}^{\infty} \mathbb{Z}$, and $x r$ $=\left(x_{n} r_{n}\right)$. For any one of the direct summands $Z_{4} e_{12} \subset \oplus_{1}^{\infty} Z_{4} e_{12} \subset R$, 
$\left(2 Z_{4} e_{12}\right)^{\perp}<<R$ is large. Also $\left(2 Z_{4} e_{11}\right)^{\perp}<<R$. Then $\mathbf{Z} R=2 Z_{4} e_{11}+$ $\oplus_{1}^{\infty} 2 Z_{4} e_{12}$ while $\mathbf{Z}_{2} R=Z_{4} e_{11}+\oplus_{1}^{\infty} Z_{4} e_{12}$ is the first row of $R$. Thus $R / \mathbf{Z} R$ contains the following countable direct sum of torsion uniform modules $\oplus_{1}^{\infty}\left(Z_{4} e_{12}+\mathbf{Z} R\right) / \mathbf{Z} R<R / \mathbf{Z} R$. The module $R / \mathbf{Z}_{2} R$ contains an essential direct sum of uniform $R$-submodules $\oplus_{1}^{\infty} \mathbb{Z}<<\Pi_{1}^{\infty} \mathbb{Z} \cong$ $R / \mathbf{Z}_{2} R$. Consequently $R$ satisfies condition (1)(i), (ii) of Theorem $\mathrm{V}$ with $\sigma=\boldsymbol{N}_{1}$.

Next counterexamples (a) and (b) show that the blanket hypothesis (i) in Theorem $\mathrm{V}$ that $R / \mathbf{Z}_{2} R$ be discrete is actually indispensable in proving $\left(2^{*}\right) \Longrightarrow(1)$.

4.3. Consider any ring $R$ with (i) $\mathbf{Z}_{2} R=0$, (ii) where $R$ has no uniform right ideals, but (iii) $R$ does contain a nontrivial direct sum of right ideals $\oplus\left\{V_{\lambda} \mid \lambda \in \Lambda\right\} \subseteq R$ with $|\Lambda|=|R|$ summands. Then $R$ satisfies 3.3(1) for $\sigma=\sigma(R)=|R|^{+}$. Consequently by Theorem III, every $\sigma(R)$-product is injective, yet condition (1) fails because $R$ contains no uniform right ideals.

CountereXample (a). The free algebra $R=F\{x, y\}$ on two noncommuting indeterminates $x$ and $y$ over any field $F$ contains no uniform right ideals, and is torsion free. If $|F| \leq \boldsymbol{\aleph}_{0}$, then $|R|=\boldsymbol{\aleph}_{0}$. Since $\oplus\left\{x^{n} y R \mid n=0,1, \ldots\right\} \leq R$, in this case $\sigma(R)=|R|^{+}=\aleph_{1}$ is the smallest possible counterexample.

Counterexample (b). For any commutative domain $F$ with identity of cardinality $|F| \leq \boldsymbol{N}_{0}$, define $R$ to be the ring $R=\Pi_{1}^{\infty} F / \oplus_{1}^{\infty} F$. The index set $[1, \omega)=\mathbb{Z}^{+} \backslash\{0\}$ can be decomposed as a union $[1, \omega)=\bigcup Y_{s}$ of infinite subsets $Y_{s} \subset \mathbb{Z}^{+}$, where $s$ ranges over an index set of cardinality $2 \boldsymbol{N}_{0}$, and $\left|Y_{s} \cap Y_{t}\right|<\infty$ is finite if $s \neq t$. Define

$$
V_{s}=\left\{x+\bigoplus_{1}^{\infty} F \mid x=\left(x_{n}\right) \in \prod_{1}^{\infty} F, \operatorname{supp} x \subseteq Y_{s}\right\} .
$$

Then $\mathbf{Z} R=\mathbf{Z}_{\mathbf{2}} R=0$, and $\sum V_{s}=\oplus V_{s}$ is a direct sum of $|R|=2^{\aleph_{0}}$ nonzero ideals. Every $\left(2^{\aleph_{0}}\right)^{+}$-product of torsion free injectives is injective, yet $R$ contains no uniform ideals.

4.4. Application of Theorems I and IV. Let $M=C \oplus D=C \oplus$ $\Pi \mathbf{E}\left(D_{\tau}\right)$ be a torsion free injective module decomposed as in 2.9. For 
every type $\tau \in \Xi$, define $\sigma(\tau)=(\tau-d R)^{+}$; and by $2.6\left(\right.$ vi) write $D_{\tau}=$ $\oplus\left\{U_{\gamma} \mid \gamma \in \Gamma(\tau)\right\}$ where $U_{\gamma}=\hat{U}_{\gamma} \in \tau$ and $|\Gamma(\tau)|=\tau-d M$. Then (i) $\mathbf{E}\left(D_{\tau}\right) \subseteq \Pi^{\sigma(\tau)}\left\{U_{\gamma} \mid \gamma \in \Gamma(\tau)\right\}$ where the latter is an injective $\sigma(\tau)$-product for a cardinal $\sigma(\tau)$ that does not depend on $M$. Hence

(ii) if $\tau-d R<\tau-d M$, then $\mathbf{E}\left(D_{\tau}\right) \neq \Pi U_{\gamma}$.

The author is grateful to his colleague L. Fuchs for his advice. The author would like to thank the referee for a most careful reading of the paper and many valuable suggestions.

\section{REFERENCES}

[AF] F. Anderson, and K. Fuller, Rings and Categories of Modules, Graduate Texts in Math. 13, Springer (1973), New York.

[B] R. Bumby, Modules which are isomorphic to submodules of each other, Archiv. Math., XVI (1965), 184-185.

[C] A. Cailleau, Une caractérisation des modules $\Sigma$-injectives, C. R. Acad. Sci. Paris, 269 (1969), 997-999.

[CR] A. Cailleau and G. Renault, Étude des modules $\Sigma$-quasi-injectifs, C. R. Acad. Sci. Paris, 270 (1970), 1391-1394.

[CF] S. Chase and C. Faith, Quotient rings and direct products of full linear rings, Math. Z., 88 (1965), 250-264.

[CJ] P. Crawley and B. Jonsson, Refinements for infinite direct decompositions of algebraic systems, Pacific J. Math., 14 (1964), 797-855.

[D1] J. Dauns, Simple modules and centralizers, T.A.M.S., 166 (1972), 457-477.

[D2] _ Prime modules, J. Reine Angew. Math., 298 (1978), 156-181.

[D3] _ Prime modules and one sided ideals, Algebra Proceedings III, University of Oklahoma, 1979, Marcel Dekker (1979), New York, pp. 41-83.

[D4] _ Uniform modules and complements, Houston J. Math., 6 (1980), 31-40.

[D5] - Sums of Uniform Modules, Advances in Noncommutative Ring Theory, Proceedings, Plattsburgh, 1981, Lecture Notes in Math. 951, Springer (1982), pp. 68-87.

[F] C. Faith, Injective Modules and Injective Quotient Rings, Marcel Dekker (1982), New York.

[FW] C. Faith and E. A. Walker, Direct sum representations of injective modules, J. Algebra, 5 (1967), 203-221.

[FU] C. Faith and Y. Utumi, Quasi-injective modules and their endomorphism rings, Arch. Math., 15 (1964), 166-174.

[Fu1] L. Fuchs, On subdirect unions. I, Acta Scient. Math., III (1952), 103-120.

[Fu2]

[G]

[GB] K. R. Goodearl and A. K. Boyle, Dimension Theory for Nonsingular Injective Modules, A.M.S. Memoir No. 177, Providence, R.I. 1976.

[GZ-H] K. R. Goodearl and B. Zimmermann-Huisgen, Boundedness of direct products of torsion modules, to appear.

[GoR] R. Gordon, Krull Dimension, A.M.S. Memoir No. 133, Providence, R.I., 1973.

[HJ] K. Hrbacek and T. Jech, Introduction to Set Theory, Marcel Dekker, Inc., Monographs in Pure and Applied Math. (1978), New York. 
[J] J. Jans, Rings and Homology, Holt, Rinehart and Winston, New York, 1964.

[K1] K. Koh, On some characteristic properties of self injective rings, P.A.M.S., 19 (1968), 209-213.

[K2] _ Quasi-Simple Modules, Lectures on Rings and Modules, Lecture Notes in Math. 246, Springer (1972), New York.

[L] J. Lambek, Lectures on Rings and Modules, Chelsea Publ. Co., New York, 1976.

[Le] A. Levy, Basic Set Theory, Perspectives in Math. Logic, Springer (1979).

[Lev] _ Unique subdirect sums of prime rings, Trans. Amer. Math. Soc., 106 (1963), 64-76.

[Lo] F. Loonstra, Essential submodules and essential subdirect products, Symposia Math., XXIII (1970), 85-105.

[M] E. Matlis, Injective modules over Noetherian rings, Pacific J. Math., 8 (1958), 511-528.

[MüR] B. Müller and S. T. Rizvi, On the decomposition of continuous modules, Canad. Math. Bull., 25 (1982), 296-301.

[SV] D. Sharpe and P. Vámos, Injective Modules, Cambridge University Press, Cambr. Tracts in Math. and Math. Phys., 62 (1972).

[S1] B. Stenström, Pure submodules, Arkiv för Mat., 7 (1967), 171-195.

[S2] _ Direct sum decomposition in Grothendieck categories, Arkiv för Mat., 7 (1968), 427-432.

[S3] _ Rings of Quotients, Comprehensive Studies in Math. 217, Springer (1975), New York.

[St] H. Storrer, On Goldman's Primary Decomposition, Lecture Notes in Math. No. 246, Springer (1972), New York.

[T1] M. Teply, Torsion free injective modules, Pacific J. Math., 28 (1969), 441-453.

[T2] _ Some aspects of Goldie's torsion theory, Pacific J. Math., 29 (1969), 447-459.

[W] R. B. Warfield, Decompositions of injective modules, Pacific J. Math., 31 (1969), 236-276.

[Z-H] B. Zimmermann-Huisgen, Direct products of torsion modules, Arch. der Math., 38 (1982), 426-431.

[Z-HZ] B. Zimmermann-Huisgen and W. Zimmerman, Classes of modules with the exchange property, to appear.

Received July 22, 1985. The author thanks the Mathematics Department of Natal University where part of this work was done. The preparation of this paper was sponsored by a Tulane University 1985 summer research grant.

TULANE UNIVERSITY

New ORLEANS, LA 70118 



\section{PACIFIC JOURNAL OF MATHEMATICS EDITORS}

\author{
V. S. VARADARAJAN \\ (Managing Editor) \\ University of California \\ Los Angeles, CA 90024 \\ Herbert Clemens \\ University of Utah \\ Salt Lake City, UT 84112 \\ R. FINN \\ Stanford University \\ Stanford, CA 94305
}

\section{HERMANN FLASCHKA}

University of Arizona

Tucson, AZ 85721

RAMESH A. GANGOLLI

University of Washington

Seattle, WA 98195

VAUGHAN F. R. JONES

University of California

Berkeley, CA 94720

ROBION KIRBY

University of California

Berkeley, CA 94720
C. C. MOORE

University of California

Berkeley, CA 94720

H. SAMELSON

Stanford University

Stanford, CA 94305

HAROLD STARK

University of California, San Diego

La Jolla, CA 92093

\section{ASSOCIATE EDITORS}
R. ARENS
E. F. BECKENBACH
B. H. NEUMANN
F. WOLF
K. YOSHIDA
(1906-1982)

\section{SUPPORTING INSTITUTIONS}

UNIVERSITY OF ARIZONA UNIVERSITY OF OREGON

UNIVERSITY OF BRITISH COLUMBIA UNIVERSITY OF SOUTHERN CALIFORNIA

CALIFORNIA INSTITUTE OF TECHNOLOGY STANFORD UNIVERSITY

UNIVERSITY OF CALIFORNIA

MONTANA STATE UNIVERSITY

UNIVERSITY OF HAWAII

UNIVERSITY OF NEVADA, RENO

UNIVERSITY OF TOKYO

NEW MEXICO STATE UNIVERSITY

UNIVERSITY OF UTAH

OREGON STATE UNIVERSITY

WASHINGTON STATE UNIVERSITY

UNIVERSITY OF WASHINGTON 


\section{Pacific Journal of Mathematics}

\section{Vol. 126, No. $1 \quad$ November, 1987}

John Dauns, Uniform dimensions and subdirect products $\ldots \ldots \ldots \ldots \ldots 1$

William B. Jacob, Quadratic forms over dyadic valued fields. I. The graded

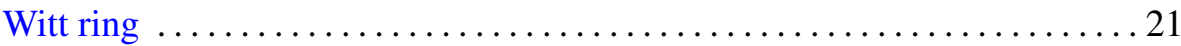

Michael R. Kelly, Minimizing the number of fixed points for self-maps of

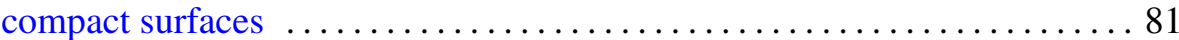

Edward Kissin, On some reflexive operator algebras constructed from two sets of closed operators and from a set of reflexive operator algebras . . 125

Robert Langlands, The Dirac monopole and induced representations . . . . . 145

David A. Stegenga and Kenneth R. Stephenson, Generic covering properties for spaces of analytic functions. II $\ldots \ldots \ldots \ldots \ldots \ldots \ldots 153$

Gerard Alan Venema, Approximating codimension two embeddings of cells 165

Peter Waksman, Determining an analytic function from its distribution of values 